\title{
Study on Construction of Safety Management Capability Model of Dangerous Goods Transportation for Aviation Logistics Enterprises
}

\author{
Ziruo Jia ${ }^{* 1, a}$, Ran Wei ${ }^{1, b}$ \\ ${ }^{1}$ School of Economics and ManagementCivil Aviation University of China Tianjin, China
}

\begin{abstract}
To clarify the mutual relations and structure attributes of safety management capability elements of dangerous goods transportation for aviation logistics enterprises, safety management capability analysis method coupled by analytic hierarchy process (AHP) and DEMATEL is proposed in this paper. Firstly, the conceptual model of safety management capability of dangerous goods transportation for aviation logistics enterprises which takes into account of emergence was established based on existing researches of safety management capability, and the composition elements were confirmed. Secondly, for the defects of traditional DEMATEL method, AHP-based and improved DEMATEL method was constructed. Finally, importance judgment matrix of factors influencing safety management capability of dangerous goods transportation for aviation logistics enterprises was gained through questionnaire inquiry. Meanwhile, the mutual relations of capability factors were confirmed through the centrality and reasons. The research result shows that, the method can be used to analyze safety management capability of dangerous goods transportation for aviation logistics enterprises and construct the structural model.
\end{abstract}

\section{Introduction}

As air transportation quantity of dangerous goods increases year by year, non-security incidents of dangerous goods transportation also increase. Due to the peculiarity of air transportation, air transportation accidents of dangerous goods will lead to very severe consequences. The major cause for accidents is that aviation logistics enterprises have insufficient safety management capability for dangerous goods transportation. Thus, it is very significant to systematically analyze composition elements and their structural attributes of safety management capability of dangerous goods transportation of aviation logistics enterprises and construct safety management capability model for improving safety management capability of dangerous goods transportation of aviation logistics enterprises and reducing accident occurrence possibility. At present, scholars have explored enterprise safety management capability in different fields and some valuable achievements have formed. Liu Tiezhong set up the structural model of safety management capability of coal mine enterprises through questionnair e survey and structural equation model.[1-3]

Liu T.z et al. proposed an optimized model of safety management capability on the basis of analyzing coal mine safety management capability model. Liu Q.1 et al. recognized the composition elements of coal mine safety management capability from the perspective of safety system engineering, and applied neural network model to evaluate safety management capability level. Li Jiangyuan established safety management capability model of airlines from four dimensions (staff, equipment, environment and system) and adopted entropy evaluation method to set up quantitative evaluation model. Guo Haidong et al. coupled maturity model and capability model to construct safety management capability measurement model of construction enterprises. In conclusion, these researches mainly focus on coal, building and aviation fields, but fail to involve dangerous goods transportation field of aviation logistics enterprises. During construction of existing safety management capability model, causal relationship among dimension elements and element effect results are not analyzed fully. Such causal relationship and effect results can be described by emergence. The fundamental objective of this study is to promote safety management capability of dangerous goods transportation of aviation logistics enterprises through analysis, control and guidance of such emergence behavior as well as utilizing action mechanism of emergence in formation of safety management capability. Therefore, the conceptual model of safety management capability of dangerous goods transportation for aviation logistics enterprises which takes into account of emergence was established based on existing researches of safety management capability, and the composition elements were confirmed. Delphi method was applied to confirm relative importance judgment matrix of elements. Besides, AHP and DEMATEL were used to establish

\footnotetext{
${ }^{*}$ Zrjia@cauc.edu.cn

bRwei@cauc.edu.cn
} 
safety management capability analysis model of dangerous goods transportation for aviation logistics enterprises. Element effect relationship and structure attribute were confirmed through analyzing the model and calculating centrality and reason of safety management capability elements. At the same time, structural model of safety management capability was established, in the hope of providing theoretical reference for aviation logistics enterprises to improve safety management capability of dangerous goods. [4-5]

\section{Methodology}

The existing definition of safety management capability concept mainly includes two aspects: essence of safety management capability, outward manifestation form of safety management capability. During defining safety management capability of dangerous goods transportation of aviation logistics enterprises, the above two aspects were also considered. First of all, the essence of safety management capability of dangerous goods transportation of aviation logistics enterprises is the ability of aviation logistics enterprises to cope with various hidden dangers in dangerous goods transportation. The outward manifestation of safety management capability of dangerous goods transportation of aviation logistics enterprises is the ability to maintain system security state. Dangerous goods transportation system of aviation logistics enterprises should include four sub-systems: infrastructure, operating personnel, organizational management and transportation environment. In conclusion, safety management capability of dangerous goods transportation of aviation logistics enterprises refers to the ability of aviation logistics enterprises to effectively organize and coordinate the four elements (infrastructure, operating personnel, organizational management and transportation environment) by comprehensively applying safety management capability of dangerous goods transportation so as to achieve safety transportation capability of dangerous goods transportation. Safety management capability of dangerous goods transportation means aviation logistics enterprises continuously accumulate Safety management capability, integrate safety knowledge and resources, adjust system element state of infrastructure, operating personnel, organizational management and transportation environment, improve transportation process safety and thus make composition elements of safety management capability of dangerous goods transportation gradually become complex in order to cope with potential safety hazards in dangerous goods transportation. Meanwhile, the composition elements of safety management capability form the new safety management capability hierarchy and structural features through mutual influence and interactions, and finally manifest as the overall control ability of aviation logistics enterprises for dangerous goods transportation safety elements.

Emergence refers to the process of forming new system traits that each element does not possess under interactions of system elements. Thus, based on analyzing the concept and connotation of safety management capability of dangerous goods transportation of aviation logistics enterprises, there are two emergence stages in the formation process of safety management capability of dangerous goods transportation. Emergence stage 1 refers to the formation of subsystem ability of composition elements of safety management capability, i.e. each subsystem element forms the ability attribute that single element does not own through interactions and mutual influence. Emergence stage 2 means element subsystems such as infrastructure, operating personnel, organizational management and transportation environment form enterprise safety management capability through coupling and coordination. According to the above analysis, the conceptual model of safety management capability of dangerous goods transportation can be set up for aviation logistics enterprises (as shown in Figure 1.).

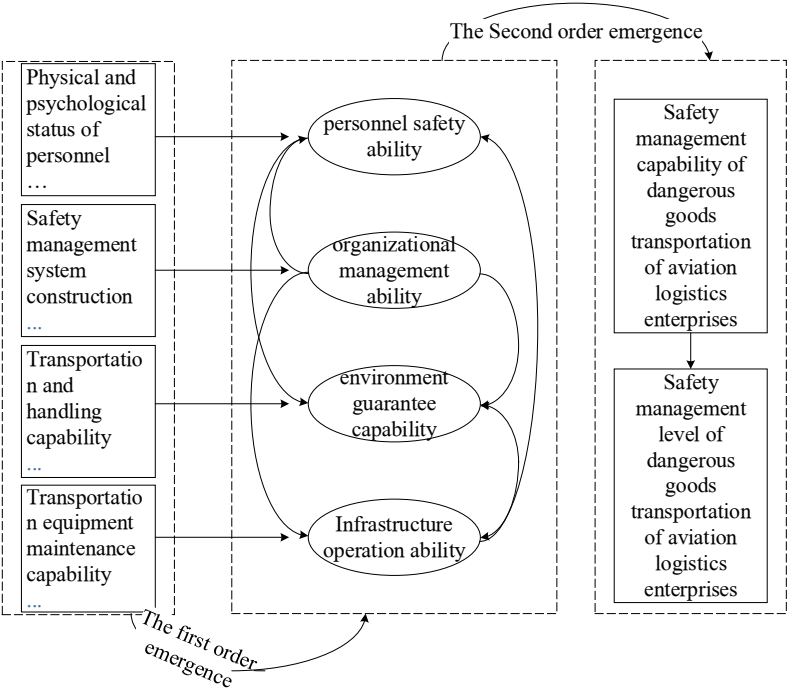

Figure 1. Conceptual model of safety management capability of dangerous goods transportation of aviation logistics enterprises

Safety management capability elements of dangerous goods transportation of aviation logistics enterprises finally emerge and form safety management capability, which is the result of mutual influence and interactions among each element. The effect relations and influence degrees of composition elements need to be analyzed. DEMATEL method which can describe mutual influence of composition elements in the complex system of safety management capability can be applied to calculate centrality and reason of composition elements of safety management capability elements of dangerous goods transportation. DEMATEL method is a complex system analysis method which achieves the purpose of analyzing system element effect relations through constructing element relation judgment matrix and conducting matrix operations. But, DEMATEL needs expert judgement information in the process of constructing the judgment matrix, while inconformity of expert knowledge and experience may easily lead to the error of judgment matrix, thus affecting the analysis result. Thus, expert judgement result consistency handling was first carried out before the judgment matrix is constructed with DEMATEL method. The improved DEMATEL method based on AHP and DEMATEL coupling was proposed. The detailed calculation steps are as follows: 
- a. Construct hierarchical structure model of safety management capability of dangerous goods transportation and establish the judgment matrix.

- b. Sort factors of safety management capability of dangerous goods transportation and carry out consistency check.

- c. Construct direct influence matrix A of safety management capability of dangerous goods transportation, gain matrix A through handling factor weight of safety management capability calculated in Step b.

$$
\mathrm{A}=\left(a_{i j}\right)=\left(w_{i} / w_{j}\right)(1)
$$

- d. Normalize direct influence matrix of safety management capability of dangerous goods transportation, standardize direct influence matrix A of safety management capability in Step b. through Formula (2) and Formula (3), and form standardized matrix $B$ of safety management capability.

$$
\begin{gathered}
\mathrm{B}=\frac{A}{x} \\
\mathrm{x}=\max \sum_{i=1}^{n}\left(\sum_{j=1}^{n} a_{i j}\right)
\end{gathered}
$$

- e. Calculate comprehensive influence matrix $\mathrm{C}$ of composition elements of safety management capability. Gain comprehensive influence matrix C of safety management capability through standardized matrix and Formula (4).

$$
\mathrm{C}=\lim _{k \rightarrow \infty}\left(B+B^{2}+B^{3}+\cdots+B^{i}\right)=B(I-X)^{-1}
$$

- f. Calculate relation parameters of composition elements of safety management capability. First of all, figure out the sum of line and row of comprehensive influence matrix $\mathrm{C}$ through Formula (5) and Formula (6) and gain influence degree $r_{i}$ of the ith composition element and being influenced degree $c_{j}$ of the ith composition element of safety management capability. Then, add and subtract influence degree $r_{i}$ and being influenced degree $c_{j}$ with Formula (7) and Formula (8) to gain the centrality $m_{i}$ and reason $\mathrm{n}_{i}$ of the ith composition element.

$$
\begin{aligned}
& r_{i}=\sum_{j=1}^{n} t_{i j}, i=1,2, \ldots n \\
& c_{j}=\sum_{i=1}^{n} t_{i j}, j=1,2, \ldots n \\
& m_{i}=r_{i}+c_{j} \\
& n_{i}=r_{i}-c_{j}
\end{aligned}
$$

Wherein, ni is the reason of the ith composition element of safety management capability. Whenn_i $>0$, the composition element $i$ is the reason element of safety management capability which can influence other safety management capability elements.

\section{Results and discussion}

\subsection{Data source and calculation}

According to the steps of safety management capability analysis model of dangerous goods transportation, subsystem analysis result and the design of concrete elements of safety management capability in the literatures, element system of safety management capability of dangerous goods transportation was established by combining Step a as shown in Figure 2.

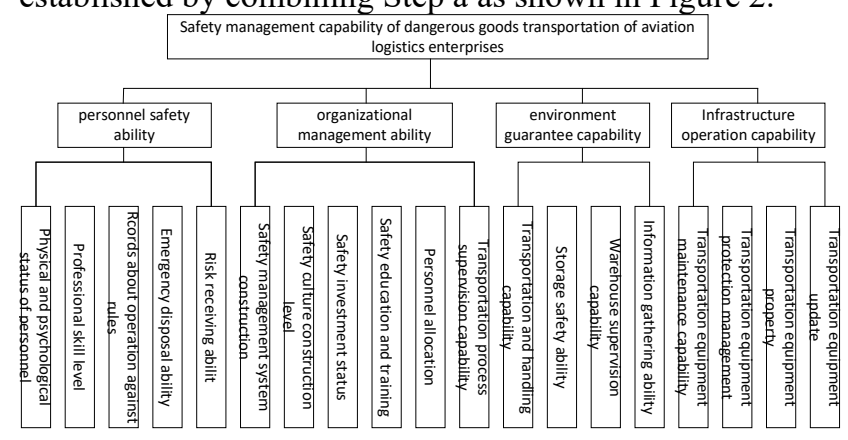

Figure 2. Element system of safety management capability of dangerous goods transportation of aviation logistics enterprises

By combining safety management capability element set of dangerous goods transportation established in Fig.2, Delphi method was used to judge and investigate capability element importance for experts in dangerous goods transportation field of aviation logistics, and the relative importance judgment matrix $\mathrm{R}=r_{i j}$ was established. Single hierarchy sorting of safety management capability elements of dangerous goods transportation was calculated according to Step b. The calculation result is as follows:

$$
\mathrm{w}=w_{i}=
$$

$(0.057,0.063,0.079,0.040,0.047,0.088,0.071,0.064,0.0$ $63,0.057,0.079,0.041,0.038,0.055,0.067,0.031,0.019,0.01$ $8,0.02) 0.057,0.065,0.078,0.041,0.048,0.087,0.074$, $0.062,0.062,0.058,0.078,0.043,0.039,0.052,0.066$, 0. 032, 0. 016, 0. 019, 0.021).

Consistency check coefficient of the above judgment matrix is $\mathrm{CR}=0.018<0.1$, which meets the requirement of consistency check. Thus, single hierarchy sorting of capability elements and Formula i can be used to figure out direct influence relation matrix A of safety management capability elements of dangerous goods transportation, as shown in Table 1.

TableI. Factors directly impact matrix

\begin{tabular}{|l|l|l|l|l|l|l|l|l|l|l|l|}
\hline & $s_{1}$ & $s_{2}$ & $s_{3}$ & $s_{4}$ & $s_{5}$ & $s_{6} \cdots s_{14}$ & $s_{15}$ & $s_{16}$ & $s_{17}$ & $s_{18}$ & $s_{19}$ \\
\hline$s_{1}$ & 1.000 & 0.903 & 0.751 & 1.440 & 1.228 & $\cdots$ & 0.891 & 1.847 & 3.689 & 3.101 & 2.811 \\
\hline$s_{2}$ & 1.092 & 1.000 & 0.821 & 1.582 & 1.353 & $\cdots$ & 0.882 & 1.847 & 3.692 & 3.117 & 2.812 \\
\hline$s_{3}$ & 1.311 & 1.200 & 1.000 & 1.904 & 1.626 & $\cdots$ & 0.991 & 2.030 & 4.061 & 3.425 & 3.091 \\
\hline$s_{4}$ & 0.690 & 0.633 & 0.527 & 1.000 & 0.855 & $\cdots$ & 1.181 & 2.433 & 4.879 & 4.103 & 3.711 \\
\hline$s_{5}$ & 0.811 & 0.741 & 0.613 & 1.172 & 1.000 & $\cdots$ & 0.620 & 1.283 & 2.563 & 2.157 & 1.950 \\
\hline$s_{6}$ & 1.468 & 1.344 & 1.114 & 2.123 & 1.811 & $\cdots$ & 0.729 & 1.501 & 2.999 & 2.525 & 2.284 \\
\hline$s_{7}$ & 1.249 & 1.141 & 0.951 & 1.803 & 1.542 & $\cdots$ & 1.319 & 2.721 & 5.439 & 4.577 & 4.142 \\
\hline$s_{8}$ & 1.047 & 0.956 & 0.796 & 1.515 & 1.293 & $\cdots$ & 1.123 & 2.311 & 4.623 & 3.896 & 3.526 \\
\hline
\end{tabular}




\begin{tabular}{|l|l|l|l|l|l|l|l|l|l|l|l|}
\hline$s_{9}$ & 1.044 & 0.956 & 0.794 & 1.511 & 1.295 & $\cdots$ & 0.940 & 1.939 & 3.877 & 3.261 & 2.951 \\
\hline$s_{10}$ & 0.979 & 0.891 & 0.750 & 1.413 & 1.209 & $\cdots$ & 0.937 & 1.935 & 3.877 & 3.261 & 2.951 \\
\hline$s_{11}$ & 1.311 & 1.200 & 1.001 & 1.901 & 1.623 & $\cdots$ & 0.877 & 1.815 & 3.624 & 3.051 & 2.761 \\
\hline$s_{12}$ & 0.721 & 0.661 & 0.553 & 1.047 & 0.895 & $\cdots$ & 1.181 & 2.433 & 4.876 & 4.103 & 3.715 \\
\hline$s_{13}$ & 0.657 & 0.599 & 0.502 & 0.953 & 0.811 & $\cdots$ & 0.651 & 1.342 & 2.688 & 2.261 & 2.047 \\
\hline$s_{14}$ & 0.875 & 0.809 & 0.669 & 1.267 & 1.082 & $\cdots$ & 0.591 & 1.217 & 2.437 & 2.051 & 1.857 \\
\hline$s_{15}$ & 1.112 & 1.019 & 0.845 & 1.613 & 1.377 & $\cdots$ & 1.000 & 1.610 & 3.251 & 2.733 & 2.467 \\
\hline$s_{16}$ & 0.541 & 0.496 & 0.411 & 0.789 & 0.669 & $\cdots$ & 2.061 & 1.000 & 4.124 & 3.480 & 3.142 \\
\hline$s_{17}$ & 0.271 & 0.249 & 0.207 & 0.391 & 0.329 & $\cdots$ & 0.481 & 1.989 & 1.000 & 1.688 & 1.530 \\
\hline$s_{18}$ & 0.321 & 0.296 & 0.245 & 0.461 & 0.398 & $\cdots$ & 0.245 & 0.503 & 0.844 & 1.000 & 0.761 \\
\hline$s_{19}$ & 0.353 & 0.321 & 0.267 & 0.513 & 0.437 & $\cdots$ & 0.298 & 0.591 & 1.190 & 0.907 & 1.000 \\
\hline
\end{tabular}

MATLAB software was used to program for calculation steps in $\mathrm{d}$ to $\mathrm{f}$ according to direct influence relation matrix of safety management capability elements of dangerous goods transportation. Then, comprehensive influence relation matrix and relation parameters of safety management capability elements of dangerous goods transportation could be obtained. The results are shown in Table 2 and Table 3.

TableII. Comprehensive influence matrix

\begin{tabular}{|c|c|c|c|c|c|c|c|c|c|c|c|}
\hline & $\mathbf{s}_{1}$ & $s_{2}$ & $\mathbf{s}_{3}$ & $\mathbf{s}_{4}$ & $\mathbf{S}_{5}$ & $s_{6} \cdots s_{14}$ & $\mathbf{s}_{15}$ & $s_{16}$ & $\mathbf{s}_{17}$ & $S_{18}$ & $s_{19}$ \\
\hline $\mathbf{s}_{1}$ & 0.047 & 0.041 & 0.035 & 0.067 & 0.058 & $\cdots$ & 0.043 & 0.089 & 0.175 & 0.147 & 0.133 \\
\hline $\mathbf{s}_{2}$ & 0.051 & 0.046 & 0.041 & 0.077 & 0.063 & $\cdots$ & 0.046 & 0.098 & 0.192 & 0.162 & 0.148 \\
\hline $\mathbf{s}_{3}$ & 0.061 & 0.059 & 0.093 & 0.091 & 0.075 & $\cdots$ & 0.054 & 0.117 & 0.231 & 0.194 & 0.174 \\
\hline $\mathbf{s}_{4}$ & 0.321 & 0.031 & 0.047 & 0.046 & 0.040 & $\cdots$ & 0.031 & 0.062 & 0.123 & 0.105 & 0.092 \\
\hline $\mathbf{s}_{5}$ & 0.040 & 0.035 & 0.054 & 0.055 & 0.047 & $\ldots$ & 0.034 & 0.072 & 0.142 & 0.125 & 0.107 \\
\hline$s_{6}$ & 0.071 & 0.063 & 0.102 & 0.103 & 0.084 & $\cdots$ & 0.062 & 0.127 & 0.258 & 0.219 & 0.199 \\
\hline $\mathbf{s}_{7}$ & 0.060 & 0.055 & 0.087 & 0.086 & 0.072 & $\cdots$ & 0.055 & 0.111 & 0.221 & 0.186 & 0.169 \\
\hline$s_{8}$ & 0.051 & 0.042 & 0.073 & 0.071 & 0.061 & $\cdots$ & 0.044 & 0.091 & 0.183 & 0.156 & 0.143 \\
\hline $\mathbf{S}_{9}$ & 0.051 & 0.043 & 0.077 & 0.072 & 0.059 & $\cdots$ & 0.043 & 0.095 & 0.187 & 0.155 & 0.147 \\
\hline$s_{10}$ & 0.048 & 0.043 & 0.068 & 0.069 & 0.057 & $\cdots$ & 0.041 & 0.085 & 0.172 & 0.144 & 0.133 \\
\hline$s_{11}$ & 0.062 & 0.057 & 0.092 & 0.093 & 0.078 & $\cdots$ & 0.055 & 0.117 & 0.233 & 0.194 & 0.175 \\
\hline$s_{12}$ & 0.033 & 0.035 & 0.051 & 0.052 & 0.042 & $\cdots$ & 0.033 & 0.065 & 0.129 & 0.109 & 0.099 \\
\hline$s_{13}$ & 0.032 & 0.028 & 0.044 & 0.043 & 0.037 & $\cdots$ & 0.027 & 0.058 & 0.117 & 0.099 & $\begin{array}{c}0.085 \\
\end{array}$ \\
\hline$s_{14}$ & 0.043 & 0.037 & 0.061 & 0.062 & 0.051 & $\cdots$ & 0.035 & 0.079 & 0.154 & 0.132 & 0.119 \\
\hline$s_{15}$ & 0.051 & 0.050 & 0.074 & 0.076 & 0.068 & $\cdots$ & 0.048 & 0.097 & 0.195 & 0.167 & 0.152 \\
\hline$s_{16}$ & 0.025 & 0.023 & 0.038 & 0.039 & 0.031 & $\cdots$ & 0.021 & 0.047 & 0.092 & 0.081 & 0.072 \\
\hline$s_{17}$ & 0.011 & 0.013 & 0.020 & 0.018 & 0.015 & $\cdots$ & 0.011 & 0.023 & 0.047 & 0.041 & $\mathbf{0 . 0 3 5}$ \\
\hline$s_{18}$ & 0.013 & 0.015 & 0.021 & 0.020 & 0.018 & $\cdots$ & 0.013 & 0.027 & 0.056 & 0.047 & 0.042 \\
\hline$s_{19}$ & 0.016 & 0.013 & 0.023 & 0.021 & 0.020 & $\ldots$ & 0.015 & 0.032 & 0.061 & 0.051 & 0.047 \\
\hline
\end{tabular}

TableIII. Factors parameters

\begin{tabular}{|c|l|l|l|l|}
\hline $\begin{array}{l}\text { Safety } \\
\text { management } \\
\text { capability } \\
\text { elements }\end{array}$ & $\begin{array}{l}\text { Influence } \\
\text { degree }\end{array}$ & Influenced degree & $\begin{array}{l}\text { Centrality } \\
\text { degree }\end{array}$ & $\begin{array}{l}\text { Cause } \\
\text { degree }\end{array}$ \\
\hline $\mathbf{s}_{\mathbf{1}}$ & 0.801 & 1.277 & 2.083 & 0.476 \\
\hline $\mathbf{s}_{\mathbf{2}}$ & 0.735 & 1.409 & 2.143 & 0.677 \\
\hline $\mathbf{s}_{\mathbf{3}}$ & 0.611 & 1.682 & 2.298 & 1.078 \\
\hline $\mathbf{s}_{\mathbf{4}}$ & 1.163 & 0.890 & 2.047 & -0.271 \\
\hline $\mathbf{s}_{\mathbf{5}}$ & 0.991 & 1.040 & 2.032 & 0.048 \\
\hline $\mathbf{s}_{\mathbf{6}}$ & 0.551 & 1.885 & 2.432 & 1.335 \\
\hline $\mathbf{s}_{\mathbf{7}}$ & 0.643 & 1.605 & 2.243 & 0.962 \\
\hline $\mathbf{s}_{\mathbf{8}}$ & 0.765 & 1.344 & 2.106 & 0.580 \\
\hline $\mathbf{s}_{\mathbf{9}}$ & 0.768 & 1.345 & 2.113 & 0.577 \\
\hline $\mathbf{s}_{\mathbf{1 0}}$ & 0.825 & 1.257 & 2.081 & 0.432 \\
\hline $\mathbf{s}_{\mathbf{1 1}}$ & 0.608 & 1.689 & 2.295 & 1.086 \\
\hline $\mathbf{s}_{\mathbf{1 2}}$ & 1.108 & 0.933 & 2.037 & -0.177 \\
\hline $\mathbf{s}_{\mathbf{1 3}}$ & 1.223 & 0.846 & 2.066 & -0.377 \\
\hline $\mathbf{s}_{\mathbf{1 4}}$ & 0.916 & 1.127 & 2.042 & 0.213 \\
\hline $\mathbf{s}_{\mathbf{1 5}}$ & 0.722 & 1.430 & 2.151 & 0.709 \\
\hline $\mathbf{s}_{\mathbf{1 6}}$ & 1.489 & 0.692 & 2.183 & -0.794 \\
\hline $\mathbf{s}_{\mathbf{1 7}}$ & 2.975 & 0.348 & 3.321 & -2.631 \\
\hline $\mathbf{s}_{\mathbf{1 8}}$ & 2.503 & 0.413 & 2.918 & -2.091 \\
\hline $\mathbf{s}_{\mathbf{1 9}}$ & 2.266 & 0.453 & -1.810 \\
\hline & & & & \\
\hline
\end{tabular}

\subsection{Model building}

In accordance with the relation parameters of safety management capability elements of dangerous goods transportation in Table 2, the reason elements mainly include $s_{1}, s_{2}, s_{3}, s_{5}, s_{6}, s_{7}, s_{8}, s_{9}, s_{10}, s_{11}, s_{14}$ and $\mathbf{s}_{15}$, while result elements mainly include $\mathbf{s}_{\mathbf{4}}, \mathbf{s}_{\mathbf{1 2}}$, $s_{13}, s_{16}, s_{17}, s_{18}$ and $s_{19}$. The result elements are influenced by reason elements. Thus, safety management subsystem capability of dangerous goods transportation forms through emergence stage 1. Finally, safety management capability of dangerous goods transportation forms through emergence stage 2. Meanwhile, in view of different degrees of effect relations among all elements, the sum of mean value and standard deviation of element influence degrees can serve as the threshold value of influence relation degree based on comprehensive influence matrix. If element influence degree is less than the threshold value, it is believed that, no effect relation exists between the two, and thus influence relation among elements forms. To sum up, reason elements and result elements served as the basic elements, subsystem 
capability elements were regarded as the interlayer, and safety management capability of dangerous goods transportation was deemed as the top layer to establish the structural model of safety management capability of dangerous goods transportation which takes into account of emergence, as shown in Figure 3.



Figure 3.Safety management capability structure model

\section{Conclusion}

The structural model of safety management capability of dangerous goods transportation of aviation logistics enterprises was analyzed from the perspective of emergence. The results show that, safety management capability of dangerous goods transportation forms finally through mutual influence and interactions among multidimension and multi-level elements as well as emergence stage 1 and emergence stage 2. Moreover, the structural model of safety management capability of dangerous goods transportation is divided into two levels: reason and result.

Traditional DEMATEL method fails to consider consistency of expert judgment information during constructing the direct influence relation matrix. For this problem, AHP-based and improved DEMATEL method was proposed in this paper to test consistency in the process of constructing direct influence relation matrix, which improves consistency of expert judgment result. At the same time, the improved DEMATEL method was used to analyze relations among safety management capability elements of dangerous goods transportation. On this basis, the structural model of safety management capability of dangerous goods transportation of aviation logistics enterprises was established from the emergence perspective, in which reason element serves as the bottom layer, result element serves as the interlayer, dimension element serves as the upper layer and management capability serves as the top layer.

\section{Acknowledgment}

R. B. G. thanks the National Soft Science Research Program (Grant: 2014GXQ4B173), the Science and Technology Program of Tianjin, China (Grant: 17ZLZDZF00100).

\section{References}

1. Fraga-Lamas, Paula; Fernandez-Carames, Tiago M.; Castedo, Luis. Towards the Internet of Smart Trains: A Review on Industrial IoT-Connected Railways. SENSORS, 17(6):1457,2017

2. Cayeux, E.; Daireaux, B.; Dvergsnes, E. W.Toward Drilling Automation: On the Necessity of Using Sensors That Relate to Physical Models. SPE DRILLING \& COMPLETION, 29(2):236-255,2014.

3. Leditschke, Jodie; Rose, Toby; Cordner, Stephen. (2015) The development of a protocol for postmortem management of Ebola virus disease in the setting of developed countries. FORENSIC SCIENCE MEDICINE AND PATHOLOGY, 11(2):2062-267,2015

4. Hamilton.The Law of Corporationsin in a Nutshell[M].West Pulishing,2001.

5. Heiko A. von der Grachtl, Inga-Lena Darkow. Scenarios for logistics srevices industry: A Delphibased analysis for 2025. International Journal of Production Economics. 127(1):46-59,2010. 